\title{
CONF-84184-.5
}

\section{MAGNETIC EXCITATION IN CUMN SPIN GLASS ALLOY}

\author{
Y. Tsunoda, N. Kunitomi, and J. W. Cable
}

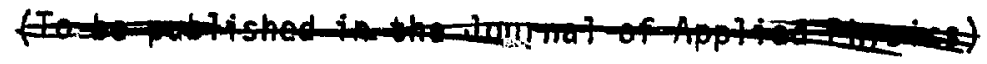

\author{
Br accoptence of this article, the \\ publisher or recipient acknowledges \\ the U.S. Gowernment's rlght to \\ rotein a nonexclusive, royal ty-free \\ license in and to any copyright \\ covaring the article.
}

\section{DISCLAIMER}

This report was prepared as an account of work sponsored by an agency of the United States Government. Neither the United States Government nor any agency thereof, nor any of their employees, makes any warranty, express or implied, or assumes any legal liability or responsibility for the accuracy, completeness, or usefulness of any information, apparatus, product, or process disclosed, or represents that its use would not infringe privately owned rights. Reference herein to any specific commercial product, process, or service by trade name, trademark, manufacturer, or otherwise does not necessarily constitute or imply its endorsement, recommendation, or favoring by the United States Government or any agency thereof. The views and opinions of authors expressed herein do not necessarily state or reflect those of the United States Government or any agency thereof.

\author{
Solid State Division \\ OAK RIDGE NATIONAL LABORATORY \\ Operated by \\ MARTIN MARIETTA ENERGY SYSTEMS, INC. \\ for the \\ U.S. DEPARTMENT OF ENERGY \\ Oak Ridge, Tennessee 37831
}


MAGNETIC EXCITATION IN CUMn SPIN GLASS ALLOY*
Y. Tsunoda and N. Kunitomi
Osaka University
Osaka, 560 Japan

and

J. W. Cable

Solid State Division, Oak Ridge National Laboratory

Oak Ridge, Tennessee 37831

\section{ABSTRACT}

The spin dynamics of a spin glass alloy $\mathrm{Cu}_{78,7} \mathrm{Mn}_{21,3}$ have been studied by neutron inelastic scattering. Measurements have been made at $20 \mathrm{~K}$, $150 \mathrm{~K}$, and $290 \mathrm{~K}$ by the constant $\mathrm{E}$ mode of operation. Magnetic excitations

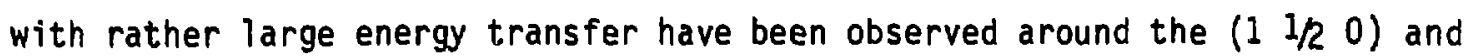
the $\left(\begin{array}{lll}1 & 1 / 2 \pm \delta & 0\end{array}\right)$ magnetic diffuse peak positions above and below the spin freezing temperature $\left(T_{f} \sim 90 \mathrm{~K}\right)$. The results indicate the existence of strong space and time correlations of the spins over the temperature range of the measurements. The experimental data are well explained by a model of dynamically fluctuating spin clusters with a broad distribution of relaxation times.

PACS numbers: $61.12 . \mathrm{Fy}, 75.40-\mathrm{s}$ 
Recent neutron scattering measurements have thrown light upon two important features of CuMn spin glasses. Murani and coworkers ${ }^{1-3}$ have studied the dynamical behavior of spin glass systems and revealed the characteristic ferromagnetic spin correlations with a broad distribution of relaxation times and a dynamical freezing process. By means of the polarization analysis technique, Cable and coworkers ${ }^{4-6}$ have observed the coexistence of two types of magnetic short-range order (MSRO); one is a modulated spin structure and the other is a ferromagnetic cluster associated with the atomic short-range order (ASRO). These are found at the (1 $1 / 2 \pm \delta 0)$ and the $(1 / / 20)$ reciprocal lattice points, respectively. Both of these observations seem to be essential for understanding the CuMn spin glass system. However, the physical relations of these properties have not yet been manifested.

We have studied the inelastic scattering of neutrons around the magnetic diffuse peak positions by using a $\mathrm{Cu}_{78,7} \mathrm{Mn}_{21.3}$ single crystal. Measurements have been performed at the $H B-3$ triple axis spectrometer installed at HFIR. The constant $E$ mode of operation has been employed at $20 \mathrm{~K}, 150 \mathrm{~K}$, and $290 \mathrm{~K}$. The freezing temperature of CuMn alloy with this Mn concentration is estimated to be $T_{f} \sim 90 \mathrm{~K}$. Most of the data were taken from the $\left(\begin{array}{lll}1 & 0 & 0\end{array}\right)$ to the $\left(\begin{array}{lll}1 & 1 & 0\end{array}\right)$ reciprocal lattice points by scanning along the $\left[\begin{array}{lll}0 & 1 & 0\end{array}\right]$ direction.

Experimental data at $20 \mathrm{~K}$ are shown in Fig. 1. The strong peak observed at $\triangle E=0 \mathrm{meV}$ is due to the superposition of the broad ASRO peak centered at ( $11 / 20)$ and the MSRO peaks. The magnetic contribution to the diffuse scattering, which is derived by subtracting the data at $290 \mathrm{~K}$ from those at $20 \mathrm{~K}$, is shown by the open circles. The magnetic diffuse scattering 
at $\Delta E=0 \mathrm{meV}$ is composed of two parts, comparatively narrow satellite diffuse peaks at $(1 / / 2 \pm \delta 0)$ and a broad peak at $(11 / 20)$; this is consistent with the previous data. 5 Weak, but still distinct, inelastic peaks are observed around both of the magnetic diffuse peak positions even though the temperature is far below the freezing temperature $\left(T-0.22 T_{f}\right)$. Figure 2 indicates the data obtained at $150 \mathrm{~K}\left(1.6 \mathrm{~T}_{f}\right)$. Elastic magnetic diffuse scattering still exists with an intensity of about one third that at $20 \mathrm{~K}$. On the other hand, the inelastic scattering intensities are markedly increased. Figure 3 shows the data at room temperature $\left(3.2 T_{f}\right)$. The elastic component of the diffuse peak is now considered to be mostly due to the ASRO, while the inelastic scattering intensities have increased even more. Longitudinal scans along the $\left[\begin{array}{lll}1 & 0 & 0\end{array}\right]$ direction passing through the (1 $1 / 2 \pm \delta 0)$ diffuse peak position have also been performed at $150 \mathrm{~K}$ and $290 \mathrm{~K}$. A single peak centered at $\left(\begin{array}{lll}1 & 1 / 2 \pm \delta & 0\end{array}\right)$ has been observed for each scan. All of the data show that the magnetic excitation peaks always locate around the position where the MSRO peaks are observed at low temperature, which indicates that a strong spatial correlation exists over the temperature range of the measurements.

We attempted to fit the line shapes of the inelastic scattering peaks under the assumption of the superposition of three Lorentzian type scattering cross sections.

$$
\frac{d^{2} a}{d \Omega d u} \propto \frac{\hbar_{u}}{e^{B \hbar \omega}-1} \frac{k^{\prime}}{k}|F(Q)|^{2} \sum_{i=1}^{3} \frac{A_{i}}{\left(Q-Q_{i}\right)^{2}+x_{i}^{2}} \frac{\Gamma_{i}}{(\hbar \omega)^{2}+\Gamma_{i}^{2}}
$$

where $Q_{1}=\left(\begin{array}{lll}1 & 1 / 2-\delta & 0\end{array}\right), Q_{2}=\left(\begin{array}{lll}1 & 1 / 2 & 0\end{array}\right)$ and $Q_{3}=\left(\begin{array}{lll}1 & 1 / 2+\delta & 0\end{array}\right), k_{j}$ is the OrnsteinZernike type inverse correlation length and $k_{1}=k_{3}$ is assumed. $A_{j}$ is 
proportional to the volume fraction associated with each peak. For simplicity the inverse relaxation time $F_{j}$ is assumed to be the same for a:1 three Lorentzians. The solid lines in Figs. 1, 2, and 3 show the calculated best fitting curves including the experimental resolution and using the parameters indicated in Table I. There ar- two points to be noticed as characteristic features of these spin glasses. (1) The inverse correlation length $k_{i}$ is almost independent of temperature for the inelastic peaks even though the line widths of the elastic diffuse peaks change with temperature. ${ }^{6}$ This leads us to a model in which the spins always jehave as clusters and the size of the fluctuating clusters does not depend much on temperature.

(2) The frequency dependence of the inelastic peaks can be well described by a constant value of $\Gamma$ rather than by the spin diffusion model with $\Gamma=r q^{2}$. Calculations based on the spin diffusion model have also been performed, but could not reproduce well the experimental line shape. This may suggest the absence of a kinematical slowing down in the spin glass system as discussed by Mezei ${ }^{7}$ and again support the model of the fluctuating spin clusters.

The previously reported properties ${ }^{6}$ of the magnetic diffuse scattering observed around $(1 / 2 \pm \delta 0)$ also give several indications that these MSRO peaks show a dynamical freezing process. (1) The temperature dependence of the diffuse peak intensities is not like a Brillouin function, but almost linear. (2) The line width of the diffuse peak does not depend on Mn concentration, but becomes broader at higher temperature. (3) The magnitude of the magnetic moment estimated from the diffuse peak intensities is smaller than that expected for a localized Mn moment in noble metals. Futhermore, in the present measurements, the relative intensities of inelastic scattering observed around the magnetic diffuse peak positions are far stronger than those 
expected for magnon scattering from usual magnetic substances. All of these properties are well understood by the model of dynamically fluctuating spin clusters with a broad distribution of relaxation times.

Murani et a $1^{1-3}$ have regarded the time correlation of spins as the

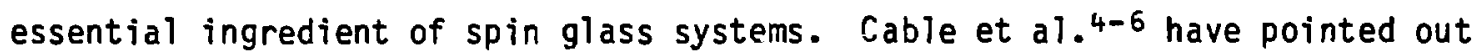
the significance of the MSRO. The present results indicate the importance of the space and time correlations of the spins and provide us the following picture of the CuMn spin glasses. At $T$ well above $T_{f}\left(T \sim 3 T_{f}\right)$, since strong inelastic scattering is observed around the MSRO peak positions, a rather large portion of the spins already couple locally and build various sizes of spin clusters. The orientations of the spins of these clusters are rotating as a whole. In the case of CuMn alloy, there are mainly two types of clusters, one with the modulated spin structure and the other with the ferromagnetin structure associated with the ASRO. As the temperature is lowered towards $T_{f}$, the interactions among the clusters gradually evolves and some portion of the clusters begins to freeze according to each local environmental condition. In the course of this process, the distribution of relaxation times becomes broader and broader as extensively discussed by Mezei et $a 1 . .^{3}$ and the elastic component of diffuse scattering increases. Below $T_{f}$, the interacting clusters may be connected into a macroscopic size. However, since each cluster freezes with random orientation, elastic scattering of neutrons still shows broad diffuse peaks even though certain clusters may grow in size in the freezing process. Even at the lowest temperature, inelastic scattering is observed around the MSRO position, indicating the existence of fluctuating clusters around their own easy axes or the persistence of nonpercolated ciusters probably due to frustration. This picture 
of the CuMn spin glass is almost the same as the percolative type model discussed by Mydosh 8 and other authors. ${ }^{7}$ The present experimental results constitute direct evidence of the validity of this model for the description of an actual spin glass system. 


\section{REFERENCES}

* Research carried out at the Oak Ridge National Laboratory under the U.S.Japan Cooperative Program in Neutron Scattering and sponsored in part by the U.S. Department of Energy under contrac1; DE-AC05-840R21400 with Martin Marietta Energy Systems, Inc.

1A. P. Murani, and J. L. Tholence, Solid State Commun. 22, 25 (1977).

2A. P. Murani, Phys. Rev. Lett. 41,1406 (1978).

${ }^{3}$ F. Mezei and A. P. Murani, J. Magn. Magn. Mater. 14, 211 (1979).

4S. A. Werner and J. W. Cable, J. Appl. Phys. 52(3), 1757 (1981).

5J. W. Cable, S. A. Werner, G. P. Felcher, and N. Wakabayashi, Phys. Rev. Lett. 49,829 (1982).

6J. W. Cable, S. A. Werner, G. P. Felcher, and N. Wakabayashi, Phys. Rev.

B 29, 1268 (1984).

7F. Mezei, J. Magn. Magn. Mater. 31-34, 1327 (1983).

8J. A. Mydosh, J. Magn. Mag. Mater. 15-18, 99 (1980). 
Table I. Lorentzian fitting parameters for $\mathrm{Cu}_{78,7} \mathrm{Mn}_{21,3}$

\begin{tabular}{|c|c|c|c|c|c|c|c|}
\hline & $\begin{array}{c}\delta \\
\left(A^{-1}\right)\end{array}$ & $A_{2} / A_{1}$ & $A_{3} / A_{1}$ & $\begin{array}{r}\kappa_{1}=\kappa_{3} \\
\left(A^{-1}\right)\end{array}$ & $\begin{array}{c}\kappa_{2} \\
\left(A^{-1}\right)\end{array}$ & $\begin{array}{c}r \\
(\mathrm{meV})\end{array}$ & $\begin{array}{r}N \div) \\
\times 10^{-3} \\
\end{array}$ \\
\hline $20 \mathrm{~K}$ & & $9 \pm 2$ & & $0.12 \pm 0.02$ & & $3.5 \pm 0.7$ & 1.10 \\
\hline $150 \mathrm{~K}$ & $\begin{array}{c}0.35 \\
\text { (fixed) }\end{array}$ & $13 \pm 1$ & $\begin{array}{c}1.30 \\
\text { (fixed) }\end{array}$ & $0.11 \pm 0.01$ & $0.38 \pm 0.04$ & $3.5 \pm 0.5$ & 0.90 \\
\hline $290 \mathrm{~K}$ & & $11 \pm 1$ & & $0.12 \pm 0.01$ & & $4.5 \pm 0.5$ & 0.71 \\
\hline
\end{tabular}

*) Normalization constant between the calculated intensitfes and the observed values. 


\section{FIGURE CAPTIONS}

Fig. 1. Experimental data at $20 \mathrm{~K}$. Solid lines show the calculated line profiles by using the fitting parameters given in Table I. Broken lines indicate the background counts estimated from the line shape fitting calculation. Open circles show the magnetic contribution of elastic diffuse scattering.

Fig. 2. Experimental data at $150 \mathrm{~K}$ and the best fitting curves. Fig. 3. Experimental data at $290 \mathrm{~K}$ and the best fitting curves. 


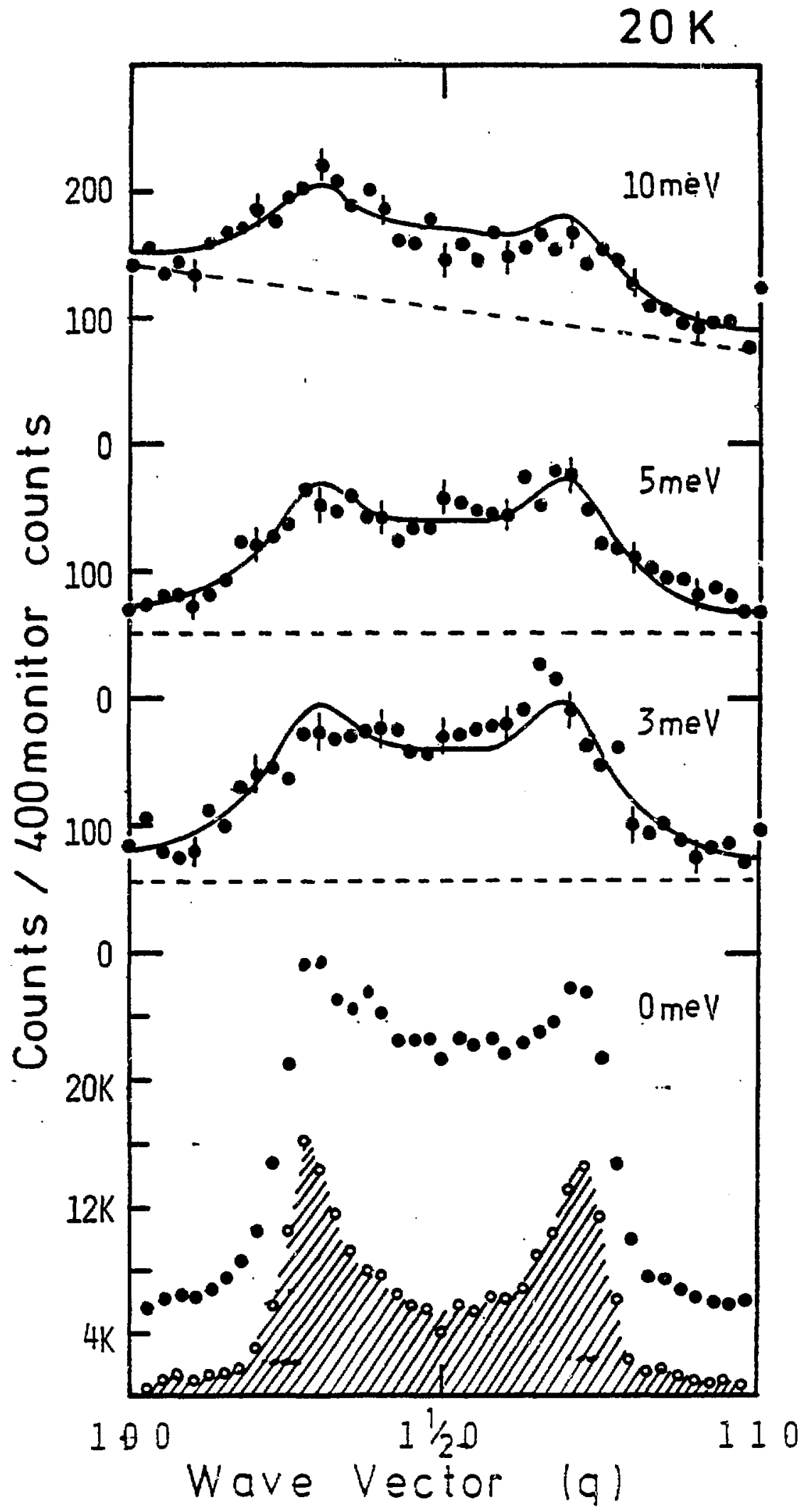




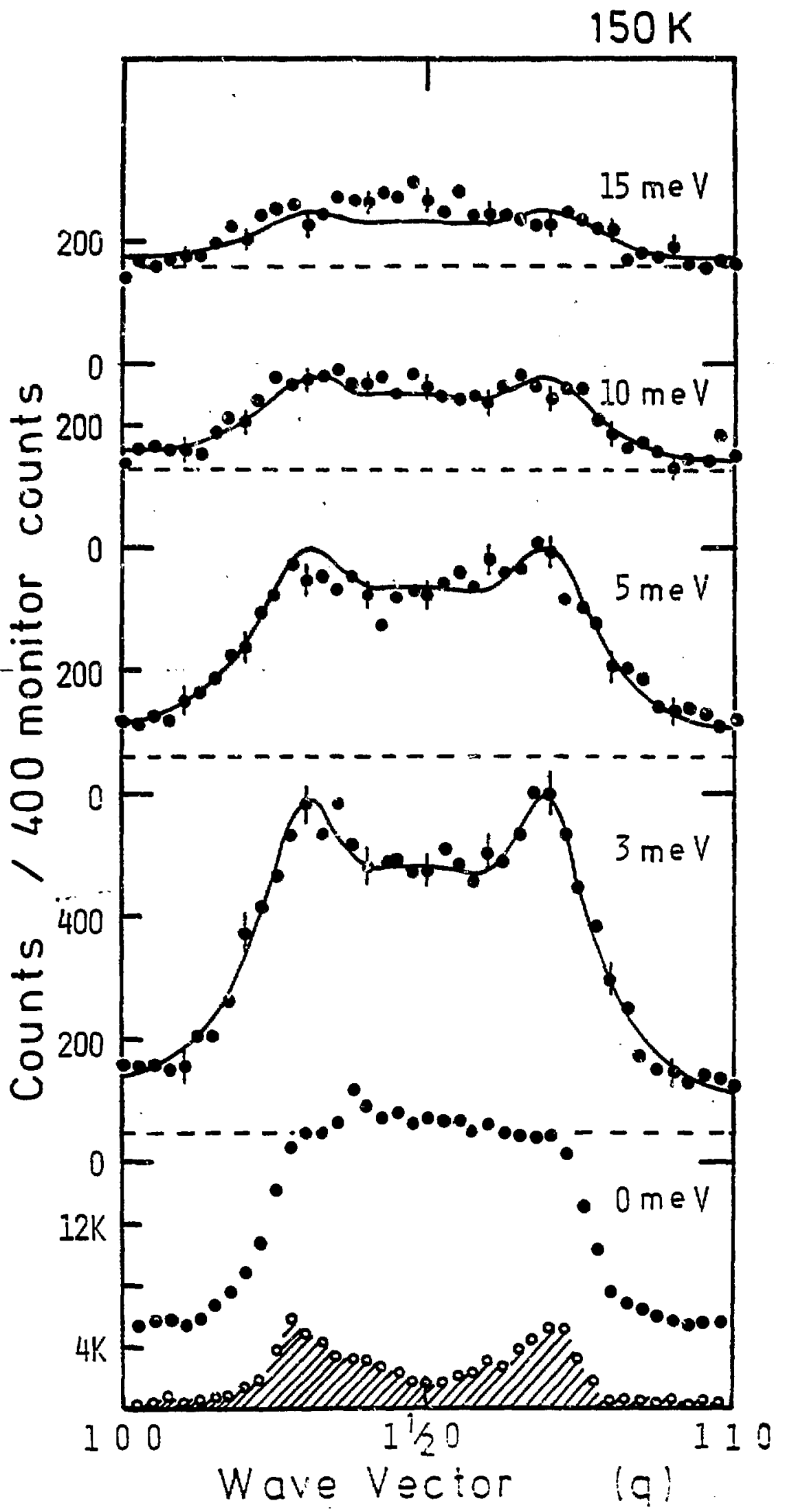

Fig. 
290K

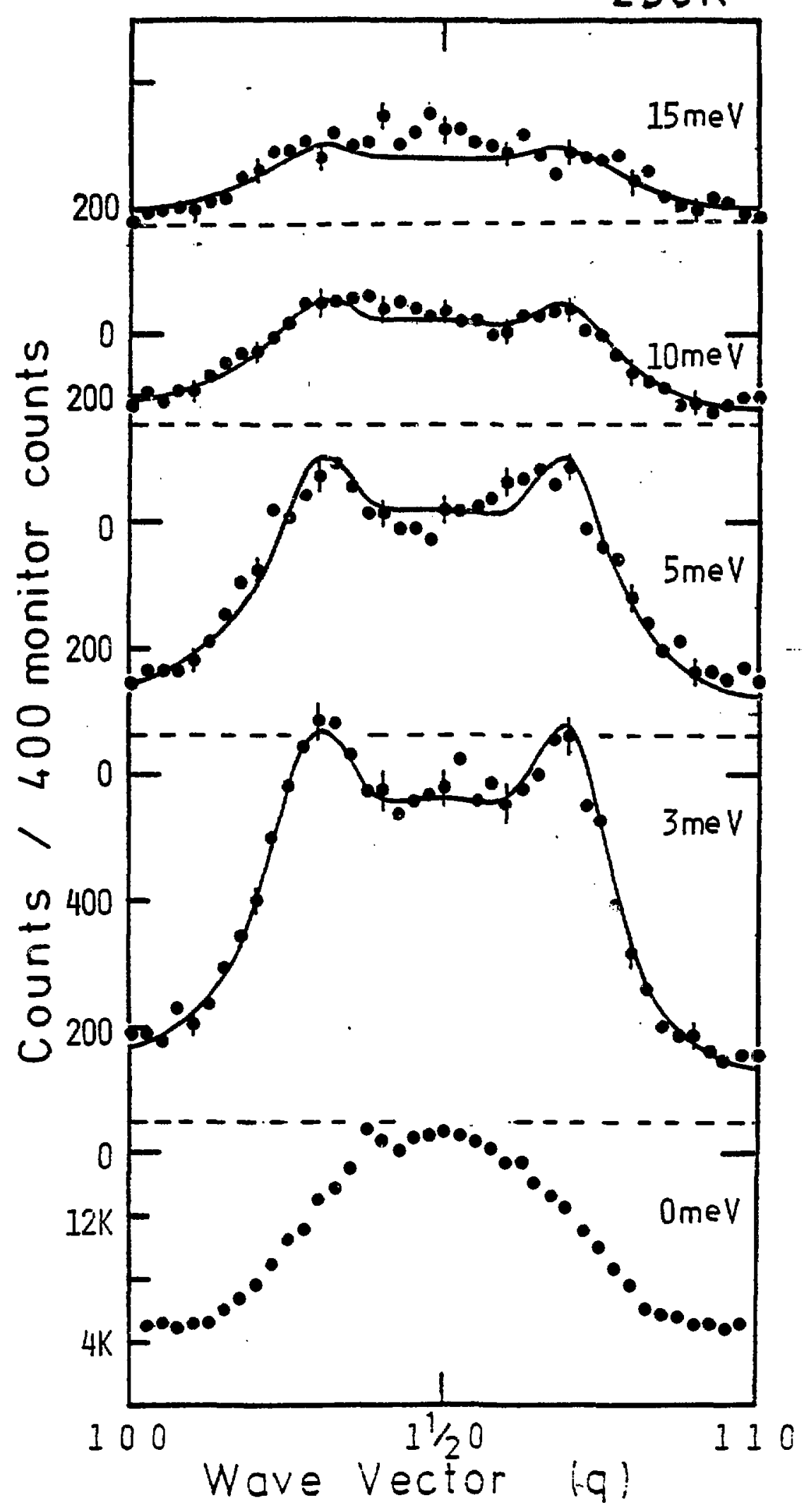

